\title{
Triple-gluon and quark-gluon vertex from lattice QCD in Landau gauge
}

\author{
A. Sternbeck ${ }^{* 1}$, P.-H. Balduf ${ }^{1,2}$, A. Kızılersü ${ }^{3}$, O. Oliveira $^{4}$, P.J. Silva ${ }^{4}$, J. Skullerud ${ }^{5}$, \\ A. G. Williams ${ }^{3}$ \\ ${ }^{1}$ Theoretisch-Physikalisches Institut, Friedrich-Schiller-Universität Jena, 07743 Jena, Germany \\ 2 Institut für Physik, Humboldt-Universität zu Berlin, 12489 Berlin, Germany \\ ${ }^{3}$ CSSM, Department of Physics, University of Adelaide, Adelaide, SA 5005, Australia \\ ${ }^{4}$ CFisUC, Department of Physics, University of Coimbra, P-3004 516 Coimbra, Portugal \\ ${ }^{5}$ Department of Mathematical Physics, National University of Ireland Maynooth, Ireland \\ E-mail: andre.sternbeckeuni-jena.de
}

\begin{abstract}
We report on preliminary results for the triple-gluon and the quark-gluon vertex in Landau gauge. Our results are based on two-flavor and quenched lattice QCD calculations for different quark masses, lattice spacings and volumes. We discuss the momentum dependence of some of the vertices's form factors and the deviations from the tree-level form.
\end{abstract}

34th annual International Symposium on Lattice Field Theory

24-30 July 2016

University of Southampton, UK

${ }^{*}$ Speaker. 


\section{Motivation}

Lattice QCD calculations are currently the prime tool for theoretical model-free studies of hadronic properties (or QCD in general) beyond the regime of perturbation theory. There are however also other nonperturbative methods which may provide a look at strong interaction physics that complements the lattice. An example are the bound-state and Dyson-Schwinger equations (DSEs) of QCD (see [1] for a recent review). Like the lattice, this approach starts from first principles, namely the partition function, but in addition it requires fixing a gauge. In principle, any physics extracted with this method is independent of the gauge, for example, masses or form factors via correspondingly defined bound-state equations. The numerical treatment, however, requires a truncation of the infinite tower of DSEs and so a truncation (resp. gauge) dependence may enter.

Let us elucidate this with an example: In a relativistic quantum field theory hadronic states appear as poles in the spectral decomposition of $n$-point Green's functions. The residues of these poles $\left(P^{2}=-M^{2}\right)$ define the wave function of the corresponding bound state, which in the case of mesons, satisfy the Bethe-Salpether (BS) equation. This equation is typically written for the amputated wave function, aka Bethe-Salpether amplitude, and describes a meson as two-particle system of a strongly interacting quark and anti-quark. It reads [1]

$$
\Gamma_{\alpha \beta}(P, p)=\int \frac{d^{4} q}{(2 \pi)^{4}} K_{\alpha \gamma, \delta \beta}(p, q, P)\left\{S\left(q_{+}\right) \Gamma(P, q) S\left(q_{-}\right)\right\}_{\gamma \delta}
$$

where $\Gamma(p, P)$ is the BS amplitude which contains all the properties of the meson. $S\left(q_{+}\right)$and $S\left(q_{-}\right)$ are the respective quark propagators and the scattering kernel $K(p, q, P)$ encodes all possible (twoparticle-irreducible) interactions between them. $P$ is the total and $p$ the relative momentum and $q_{ \pm}=q \pm P / 2$. If $S$ and $K$ were known for all momenta, a solution of Eq. (1.1) would yield $\Gamma(p, P)$ from which masses, form factors and the like can be extracted. In fact, the masses of ground and excited states are given by all $P_{i}=-M_{i}^{2}$ for which $\Gamma\left(q, P_{i}\right)$ is a solution to Eq. (1.1).

In practice, however, neither $S$ nor $K$ are fully available. $S$ satisfies a DSE which itself needs the gluon propagator and the quark-gluon vertex as input. Both satisfy their own DSEs which involve other $n$-point functions. A truncation of the DSEs and the scattering kernel $K$ is thus needed.

A popular truncation is the rainbow-ladder (RL) truncation. It substitutes the gluon propagator by an effective propagator and reduces the quark-gluon vertex to its tree-level form. Also the scattering kernel $K$ is reduced to one (effective) gluon exchange. This often is sufficient for ground states which are found to be mostly only sensitive to the integrated scattering kernel, but observables such as excited states typically require a beyond rainbow-ladder treatment and this is where lattice results of the quark-gluon vertex can provide important input ${ }^{1}$. Since the DSE of the quark-gluon vertex contains the triple-gluon vertex, lattice results for both provides additional information how to systematically improve the truncation of the quark-gluon-vertex DSE.

Such lattice calculations are demanding, because continuum extrapolated results are needed for the many different form factors of the vertex functions. To this end, one needs good control over finite volume and lattice discretization effects. In particular the latter can be challenging due to the notorious hypercubic lattice artifacts.

\footnotetext{
${ }^{1}$ I thank G. Eichmann for briefing me about the current status.
} 
Table 1: Summary of our $N_{f}=0,2$ gauge field ensembles. The physical parameters for the $N_{f}=2$ en-

\begin{tabular}{lcccccr}
\hline \hline no. & $\beta$ & $\kappa$ & $L_{s}^{3} \times L_{t}$ & $a[\mathrm{fm}]$ & $m_{\pi}[\mathrm{MeV}]$ & \#configs. \\
\hline III & 5.20 & 0.13596 & $32^{3} \times 64$ & 0.08 & 280 & 900 \\
IV-a & 5.29 & 0.13620 & $32^{3} \times 64$ & 0.07 & 422 & 900 \\
IV-b & 5.29 & 0.13632 & $32^{3} \times 64$ & 0.07 & 295 & 908 \\
IV-c & 5.29 & 0.13632 & $64^{3} \times 64$ & 0.07 & 290 & 750 \\
V & 5.40 & 0.13647 & $32^{3} \times 64$ & 0.06 & 426 & 900 \\
\hline IV-q & 6.16 & - & $32^{3} \times 64$ & 0.07 & - & 1000 \\
II-q & 5.70 & - & $48^{3} \times 96$ & 0.17 & - & 1000 \\
I-q & 5.60 & - & $72^{3} \times 72$ & 0.22 & - & 699 \\
\hline \hline
\end{tabular}

Here we present the first steps of a long-term project. Our calculations were performed on the $N_{f}=2$ gauge field ensembles of the RQCD collaboration and, in addition, on three quenched ensembles provided by us. Lattice spacings and volumes were chosen such that a comparison with the unquenched data is possible and that (for the triple-gluon vertex) low momenta are reached (see Table 1 for details). All ensembles were fixed to Landau gauge before taking Monte-Carlo data for the required two- and three-point Green's functions.

\section{Lattice results for the triple-gluon vertex}

The Green's functions one needs for an analysis of the triple-gluon vertex are the gluon propagator $D_{\mu \nu}^{a b}(p)$ and the triple-gluon Green's function

$$
G_{\mu v \rho}^{a b c}(p, q)=\left\langle A_{\mu}^{a}(p) A_{v}^{b}(q) A_{\rho}^{c}(k)\right\rangle_{U} \quad \text { with } \quad k \equiv-(p+q) .
$$

To leading order in the lattice spacing, the gluon fields $A_{\mu}^{a}(p)$ are Fourier-transformed functions of the gauge-fixed link variables $U_{x \mu}$ :

$$
A_{\mu}^{a}(p)=\frac{1}{\sqrt{V}} \sum_{x} e^{i p x} A_{\mu}^{a}(x) \quad \text { with } \quad A_{\mu}^{a}(x) \equiv \frac{2}{a g_{0}} \mathfrak{I m} \operatorname{Tr} T^{a} U_{x \mu}
$$

We have calculated these Green's function for the subset of (discrete) momenta with $p^{2}=q^{2}$. To increase the signal-to-noise ratio we averaged our Monte-Carlo data over equal $|p|=|q|$ and also over nearby momenta before performing a time-series analysis. For the "momentum smoothing" we have tried different bin sizes $\Delta=\operatorname{abs}\left(\left|p_{2}\right|-\left|p_{1}\right|\right)=\pi /$ an with $n=128,64,32,16$ and found that already $a \Delta=\pi / 128$ results in a significant improvement of the signal-to-noise ratio.

The tensor structure of the triple-gluon vertex is described by 14 form factors (see, e.g., [3]). We are interested in the transverse part, which is accessible on the lattice and also is the interesting part in the Landau gauge. To the author's knowledge the full transverse part has never been addressed in a lattice study; only the projection of the vertex (where the color sum is implied)

$$
G_{1}(p, q)=\frac{\Gamma_{\mu \nu \rho}^{(0)}}{\Gamma_{\mu \nu \rho}^{(0)}} \frac{G_{\mu v \rho}(p, q, p-q)}{D_{\mu \lambda}(p) D_{\nu \sigma}(q) D_{\rho \omega}(p-q) \Gamma_{\lambda \sigma \omega}^{(0)}}
$$

on its tree-level form $\Gamma^{(0)}(p, q)$ has been analyzed for $S U(2)$ and $S U(3)$ Yang-Mills theories [4-6]. Those studies we complement here with quenched and unquenched data for both $G_{1}$ and the transverse form factors. For the latter we use the Bose-symmetric form of the transversely-projected 

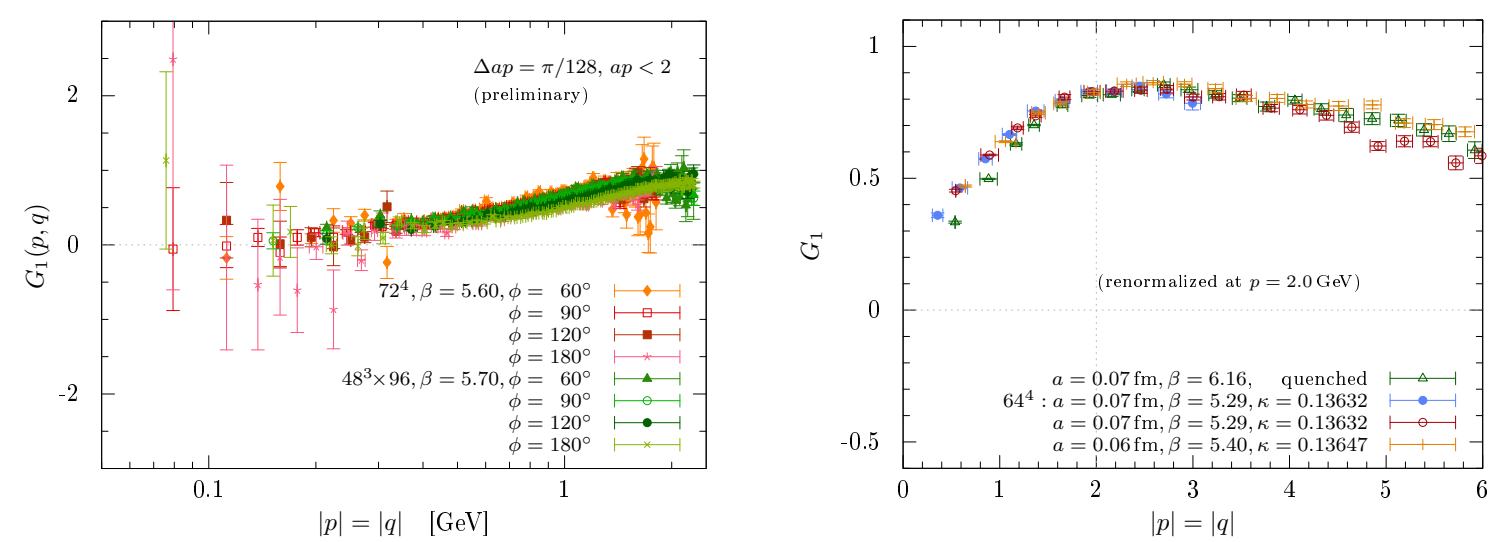

Figure 1: $G_{1}(p, q)$ versus $|p|=|q|$. Left: quenched data at low momentum for different angles $\phi=\varangle(p, q)=60^{\circ}, 90^{\circ}, 120^{\circ}$ and $180^{\circ}$. Right: for a fixed angle $\phi=90^{\circ}$ but for different quark masses and lattice spacings, and for a quenched calculation. Data for nearby-momenta has been averaged. The bin size is $a \Delta=\pi / 128$ in the left plot and $a \Delta=\pi / 32$ in the right.

triple-gluon vertex [7]

$$
\Gamma_{\mu v \rho}^{T}(p, q)=\sum_{i=1}^{4} F_{i}\left(p^{2}, q^{2}, \phi\right) \tau_{i}^{\mu v \rho}(p, q)
$$

where the four transverse form factors $F_{i}$ are functions of $p^{2}, q^{2}$ and the angle $\phi$ between $p$ and $q$. The corresponding tensor base vectors $\tau_{i}$ can be found in [7].

Data for $G_{1}$ versus $|p|=|q|$ is shown in Fig. 1. The left panel shows quenched data at low momentum for different angles $\phi$. The right panel compares quenched with unquenched data at a fixed angle $\phi=90^{\circ}$ but for different lattice spacings and quark masses. The momentum bin size is $a \Delta=\pi / 128$ for the data on the left panel and $a \Delta=\pi / 32$ on the right. To allow for a better comparison of the momentum dependence, the data points in the right panel have been renormalized relatively to each other at $\mu=2 \mathrm{GeV}$. The data in the left panel were not renormalized. There the focus is on the "zero-crossing" which was seen by others [4-6]. In fact, two recent lattice studies claim numerical evidence for $G_{1}$ crossing zero at about $|p|=100 \ldots 200 \mathrm{MeV}$ in [5] and $|p|=220 \ldots 260 \mathrm{MeV}$ in [6]. Some of our data points between $|p|=100 \ldots 300 \mathrm{MeV}$ have negative values for $G_{1}$ (e.g., for $\phi=60^{\circ}$ and $\phi=180^{\circ}$ ), but there are also several points with positive $G_{1}$. With respect to the rather large error bars, at the moment we can confirm $G_{1}$ touches zero at about $|p|=100 \ldots 200 \mathrm{MeV}$, but there is no clear numerical evidence yet for a divergence at small momenta. Perhaps future studies at much smaller momenta will answer this. A more detailed discussion will follow in [8].

Looking at the right panel of Fig. 1 we see quenching effects on the triple-gluon vertex are rather small. With the renormalization point set at $\mu=2 \mathrm{GeV}$ we see the quenched data points below $|p|=2 \mathrm{GeV}$ fall off more rapidly towards smaller $|p|$ than the unquenched. This is consistent with what has been seen in a recent DSE study of this vertex [9] assuming that $F_{1} \approx G_{1}$. As mentioned, we also looked at the four transverse form factors $F_{i=1, \ldots, 4} . F_{4}$ is zero in our kinematic, but the other three can be extracted. It turns out that $F_{1}$ by far is the dominant form factor, while $F_{2}$ and $F_{3}$ are rather small in comparison. The momentum dependence of $F_{1}$ thus roughly mirrors that 
of $G_{1}$. A detailed analysis will follow [8].

\section{Lattice results for the quark-gluon vertex}

The tensor structure of the quark-gluon vertex (in the continuum) consists of 12 independent vectors. In analogy to the Ball-Chiu decomposition of its QED counterpart, it is often written as the sum of a term satisfying the Slavnov-Taylor identity (STI) and another term which is transverse to the gluon momentum $k$. That is, $\Gamma_{\mu}(p, k)=\Gamma_{\mu}^{S T}(p, k)+\Gamma_{\mu}^{T}(p, k)$ with $k_{\mu} \Gamma_{\mu}^{T}=0$ and

$$
\Gamma_{\mu}^{S T}(p, k)=\sum_{i=1 \ldots 4} \lambda_{i}(p, k) L_{i \mu}(p, k) \quad \text { and } \quad \Gamma_{\mu}^{T}(p, k)=\sum_{i=1 \ldots 8} \tau_{i}(p, k) T_{i \mu}(p, k) .
$$

We consider here only the form factors $\lambda_{1}, \lambda_{2}$ and $\lambda_{3}$. The corresponding base vectors read [10] (all momenta are considered as outgoing)

$$
L_{1 \mu}(p, k)=\gamma_{\mu}, \quad L_{2 \mu}(p, k)=-(2 \not p+\not k)(2 p+k)_{\mu} \quad \text { and } \quad L_{3 \mu}(p, k)=-i(2 p+k)_{\mu} .
$$

$L_{4 \mu}$ and the remaining (transverse) base vectors $T_{i \mu}$ can be found in [10]. The $T$ 's are all zero for the soft-gluon kinematic $(k=0)$ which we consider here throughout. In this kinematic also $\lambda_{4}(p, 0)=0$ due to the STI and the other three $\lambda_{i}$ are functions of $p^{2}$ only, i.e., $\lambda_{i}(p, 0)=\lambda_{i}\left(p^{2}, 0\right)$.

On the lattice the tensor structure is actually even more complex due to the finite lattice spacing which breaks $O(4)$ invariance. For simplicity though, we will restrict ourselves to the continuum form, but consider lattice tree-level corrections to $L_{1 \mu}$ (resp. $\lambda_{1}$ ). This will help us to see where lattice discretisation effects are significant.

In QED, the $\lambda_{i}$ 's for the fermion-photon vertex are functions of the fermion propagator alone. This is due to the Ward-Takahashi identity. In QCD, however, the STI for the quark-gluon vertex also contains the ghost dressing function and the quark-ghost scattering kernel. A non-perturbative determination of the $\lambda_{i}$ 's for the quark-gluon vertex thus requires a calculation of 2- and 3-point functions. These are the quark propagator $S(p)$, the gluon propagator $D_{\mu v}(k)$ and the quark-antiquark-gluon 3-point function $V_{\mu, \alpha \beta}^{c, i j}=\left\langle\bar{\psi}_{\alpha}^{i} \psi_{\beta}^{j} A_{\mu}^{c}\right\rangle$ in momentum space. The form factors one gets then from the relation between vertex and 2- and 3-point functions:

$$
V_{\mu, \alpha \beta}^{c, i j}(p, k)=\Gamma_{\lambda, \sigma \rho}^{d, r s}(p, k) \cdot S_{\sigma \alpha}^{i r}(p) \cdot D_{\mu \lambda}^{c d}(k) \cdot S_{\rho \beta}^{j s}(q) \quad \text { with } \quad q=-(p+k) .
$$

We calculated $S, D$ and $V$ on all $32^{3} \times 64$ ensembles listed in Table 1. For the calculation of the quark propagator we averaged over four point sources and to reduce off-shell $O(a)$ effects we used the "rotated quark propagator" of $[11,12]$ with $b_{q}$ and $c_{q}$ set to their tree-level values. For the quenched ensembles we followed a partially quenched approach and set $\kappa=0.134$ and $c_{S W}=1.64$. According to [13] this should correspond to a pion mass value of $m_{\pi} \gtrsim 800 \mathrm{MeV}$.

In Fig. 2 we show some preliminary data for $\lambda_{1}\left(p^{2}, 0\right), \lambda_{2}\left(p^{2}, 0\right)$ and $\lambda_{3}\left(p^{2}, 0\right)$. The data are from three calculations with similar lattice spacings $(a \simeq 0.07 \mathrm{fm})$ but different quark mass values. The corresponding ensembles are listed as IV-a, IV-b and IV-q in Table 1. Our results from the lightest ensembles IV-b, that is for a pion mass of about $m_{\pi}=295 \mathrm{MeV}$ [2], are the green squares in Fig. 2. Red triangles refer to the heavier unquenched ensemble (IV-a, $m_{\pi}=422 \mathrm{MeV}$ ), while blue circles are for the partially-quenched data (IV-q) with $m_{\pi} \gtrsim 800 \mathrm{MeV}$. The points have not been renormalized to visualize differences in the bare data. 

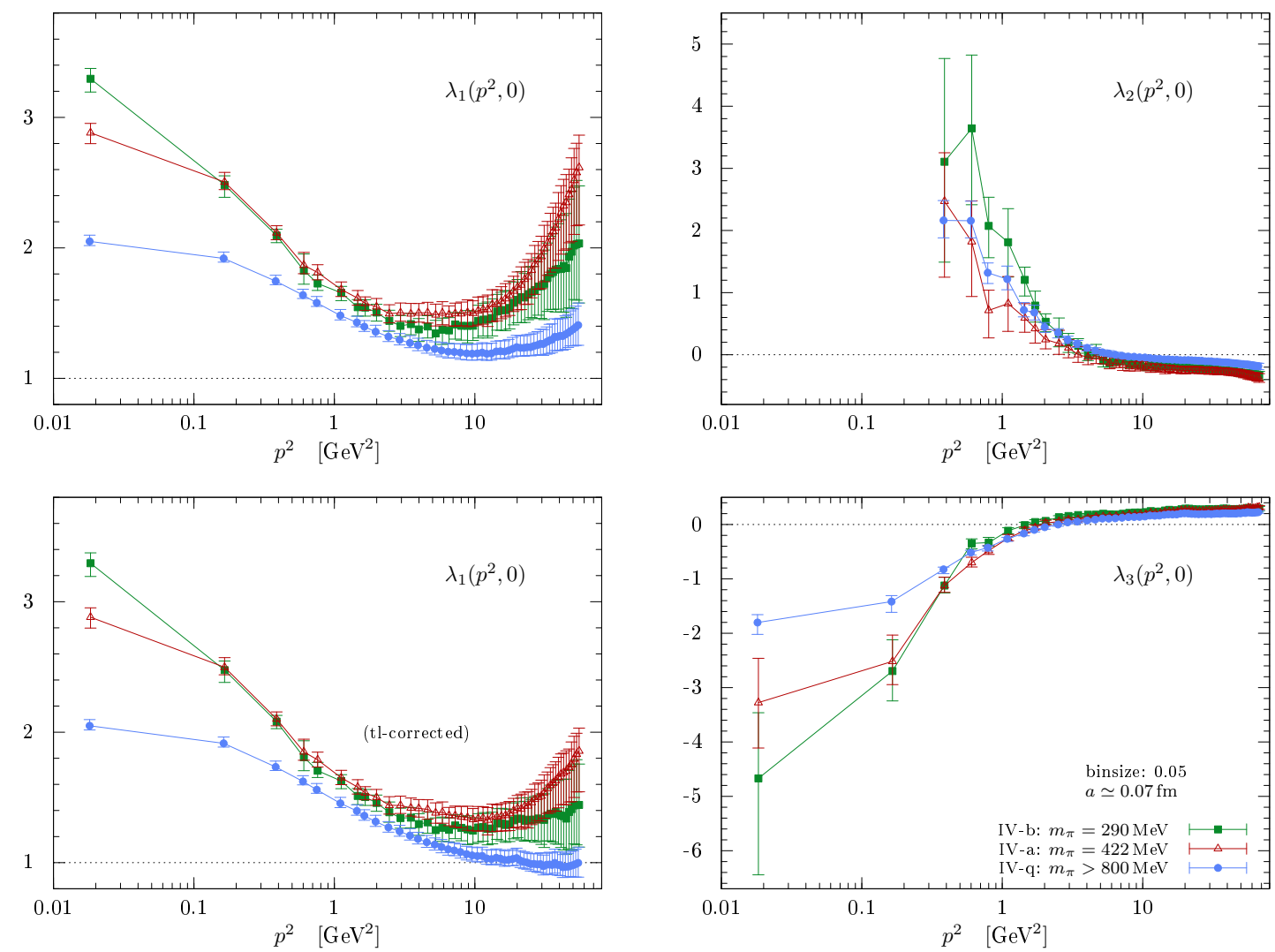

Figure 2: $\lambda_{1}\left(p^{2}, 0\right), \lambda_{2}\left(p^{2}, 0\right)$ and $\lambda_{3}\left(p^{2}, 0\right)$ from three calculations at about the same lattice spacing ( $a \simeq 0.07 \mathrm{fm}$ ) but for different bare quark masses including a quenched simulation (IV-q). The legend in the bottom right panel applies to all plots. Shown are the unrenormalized data. The bottom left panel show the corresponding lattice tree-level corrected data for $\lambda_{1}$.

Looking first at the unquenched data (squares and triangles), we see that the quark mass effects for $\lambda_{2}$ and $\lambda_{3}$ are small for high momenta but they grow towards lower $p^{2}$. For $\lambda_{1}$, on the other hand, the quark mass affects the behavior at high momentum, while at intermediate momentum the effect is small. Specifically, the two unquenched data sets for $\lambda_{1}$ match between $p^{2}=0.1 \mathrm{GeV}^{2}$ and $1 \mathrm{GeV}^{2}$, but below and above quark mass effects are seen.

Comparing the quenched and unquenched data sets for $\lambda_{1}$ and $\lambda_{3}$, we see the quenched sets have a slightly flatter momentum dependence than the unquenched. We have checked that this cannot be compensated through a simple (multiplicative) renormalization. For $\lambda_{2}$, quenched and unquenched data more or less agree. There a renormalization of the quenched data relative to the unquenched points at large $a^{2} p^{2}$ would result in a stronger momentum dependence for small $p^{2}$.

For $\lambda_{1}$ we also have lattice tree-level corrected results. By comparing corrected to uncorrected results we see no difference in the data for $\lambda_{1}$ below $a^{2} p^{2}=1$. Above $a^{2} p^{2}=1$, however, there is a clear difference and this difference also grows with $a^{2} p^{2}$. The tree-level correction brings the data closer to what is expected from perturbation theory. Our correction is not perfect and leaves room for further improvements but it suggests the strong rise of $\lambda_{1}\left(p^{2}, 0\right)$ towards larger $p^{2}$ is a lattice artifact. Note in [14] it was argued $\lambda_{1}\left(p^{2}, 0\right)$ should grow with $p^{2}$, but given the effect of the 
tree-level corrections one should be skeptical if our data will eventually confirm that. It certainly deserves further study.

Our results for $\lambda_{1}, \lambda_{2}$ and $\lambda_{3}$ below $a^{2} p^{2}=1$, that is for momenta $p^{2}<5 \mathrm{GeV}^{2}$, show no significant discretization effects. Apart from volume effects, which we expect for our lowest momentum, we are thus confident our data resemble to a good approximation the correct non-perturbative behavior of $\lambda_{1}, \lambda_{2}$ and $\lambda_{3}$ between $p^{2}=0.1 \ldots 5 \mathrm{GeV}^{2}$. In this regime we see that all three form factors show a strong deviation from the tree-level form below $p^{2}=1 \mathrm{GeV}^{2}: \lambda_{1}$ rises towards $p \rightarrow 0$ but less strong than $\lambda_{2} ; \lambda_{3}$ falls below zero towards $p \rightarrow 0$. The value of the quark mass seems to have an effect on the slope; for $\lambda_{2}$ the effect is more pronounced than for $\lambda_{1}$ and $\lambda_{3}$. Above $p^{2}=2 \mathrm{GeV}^{2}$, on the other hand, $\lambda_{1}, \lambda_{2}$ and $\lambda_{3}$, are rather close to their perturbative form. This finding may shed some light on the partial success and failure of the rainbow-ladder truncation, where only $\lambda_{1}$ is considered (modeled) but $\lambda_{2}$ and $\lambda_{3}$ are ignored.

\section{Summary}

We have presented first (preliminary) results for some of the many form factors of the triplegluon and quark-gluon vertex of QCD in Landau gauge. The data are the result of two separate projects $[8,15]$ which however base on the same set of $N_{f}=2$ ensembles of the RQCD collaboration and a few additional quenched ensembles. We have touched some issues addressed in the recent literature and gave a first impression what the momentum behavior looks like. A detailed analysis will be reported in due course.

Calculations were performed on the Cray system of the North-German Supercomputing Alliance (HLRN project bep00046) and the OMEGA cluster at the FSU Jena. We regret the sudden death of M. MüllerPreußker who collaborated with us on this project. We thank the RQCD collaboration for giving us access to their $N_{f}=2$ gauge field configurations. P.J. Silva was supported by FCT (grant SFRH/BPD/109971/2015).

\section{References}

[1] G. Eichmann et al., Prog. Part. Nucl. Phys. 91, 1 (2016), arXiv:1606.09602 [hep-ph] .

[2] G. S. Bali et al., Phys. Rev. D90, 074510 (2014), arXiv:1408.6850 [hep-lat] .

[3] J. A. Gracey, Phys. Rev. D84, 085011 (2011), arXiv:1108.4806 [hep-ph] .

[4] A. Cucchieri, A. Maas, and T. Mendes, Phys. Rev. D77, 094510 (2008), arXiv:0803.1798 [hep-lat] .

[5] A. Athenodorou et al., Phys. Lett. B761, 444 (2016), arXiv:1607.01278 [hep-ph] .

[6] A. G. Duarte et al., Phys. Rev. D94, 074502 (2016), arXiv:1607.03831 [hep-lat] .

[7] G. Eichmann, R. Williams, et al., Phys. Rev. D89, 105014 (2014), arXiv:1402.1365 [hep-ph] .

[8] P.-H. Balduf and A. Sternbeck, In preparation.

[9] R. Williams et al., Phys. Rev. D93, 034026 (2016), arXiv:1512.00455 [hep-ph] .

[10] J. Skullerud and A. Kizilersu, JHEP 09, 013 (2002), arXiv:hep-ph/0205318 [hep-ph] .

[11] J. I. Skullerud and A. G. Williams, Phys. Rev. D63, 054508 (2001), arXiv:hep-lat/0007028 [hep-lat] .

[12] J. Skullerud et al., Phys. Rev. D64, 074508 (2001), arXiv:hep-lat/0102013 [hep-lat] .

[13] M. Göckeler et al., Phys. Rev. D57, 5562 (1998), arXiv:hep-lat/9707021 [hep-lat] .

[14] A. C. Aguilar et al., (2016), arXiv:1610.06158 [hep-ph] .

[15] A. Kizilersü et al., Work in progress 